\title{
DEPREDACIÓN DE NINIA DIADEMATA (SERPENTES: COLUBRIDAE) POR UNA ARAÑA VIUDA NEGRA (LATRODECTUS MACTANS) PREDATION OF NINIA DIADEMATA (SERPENTES: COLUBRIDAE) BY BLACK WIDOW SPIDER (LATRODECTUS
}

\section{MACTANS)}

\author{
ANGEL EdUARDo CARRASCO-GONZÁLEZ ${ }^{1 *}$ \& RICARDo SERNA-LAGUNES ${ }^{1}$ \\ ${ }^{1}$ Laboratorio de Bioinformática y Bioestadística, Facultad de Ciencias Biológicas y Agropecuarias. Universidad Veracruzana. Calle Josefa Ortiz de \\ Dominguez s/n. Col. Centro, Peñuela. Amatlán de los Reyes, C.P 94950, Veracruz, México. \\ *Correspondencia:angeleduardo1723@hotmail.com
}

Abstract.- Predation of herpetozoans by spiders has been reported in tropical climate zones in Central Veracruz. However, this is the first report of predation of the collared coffee snake (Ninia diademata) by a black widow spider (Latrodectus mactans; ) in central Veracruz in a temperate climate. This information is important to increase the knowledge of the multitrophic interactions in the ecosystems that occur in the High Mountains Region, Veracruz, Mexico.

Keywords.- Coffee snake, Diet, Prey, Therediidae.

Resumen.- La depredación de herpetozoos por arañas se ha reportado en zonas de clima tropical en el centro de Veracruz. Sin embargo, este es el primer reporte de depredación de la culebra cafetalera de collar (Ninia diademata) por una araña viuda negra (Latrodectus mactans; +) en el centro de Veracruz en un clima templado. Esta información es importante para incrementar el conocimiento de las interacciones multitróficas en los ecosistemas que ocurren en la Región de Las Altas Montañas, Veracruz, México.

Palabras clave.- Culebra cafetalera, Dieta, Presa,Therediidae.

Contribuir al conocimiento de la relación depredador-presa entre arácnidos-herpetozoos ayuda a comprender la estructura de las redes tróficas y la dinámica entre las comunidades bióticas (Gaiarsa et al., 2012). Por ejemplo, se han observado arañas alimentándose de especies de serpientes juveniles con hábitos nocturnos (Aguilar-López et al., 2014).

Latrodectus mactans (Fabricius, 1775), es una especie conocida comúnmente en México como viuda negra, de la familia Theridiidae (grupo de arañas tejedoras), probablemente nativa de Norteamérica e introducida en Sudamérica y Asia (World Spider Catalog, 2019); son arácnidos depredadores oportunistas estacionarios: la técnica de caza aplicada consiste en formar una red y esperar a que las presas queden atrapadas en esta (Nyffeler, 1999). Las telarañas que construyen suelen ser irregulares, brillantes y consistentes. Recientemente son arácnidos que se han adaptado a vivir en zonas urbanas en sitios oscuros, frescos y secos; esta especie presenta dimorfismo sexual, es decir, el tamaño de los machos no sobrepasa los $10 \mathrm{~mm}$, mientras que las hembras miden entre 30 y $40 \mathrm{~mm}$ (Redtox, 2020). La dieta de las arañas del género Latrodectus es muy variada, ya que incluye un amplio rango de artrópodos como hormigas (Nyffeler et al., 1988), ciempiés (Rosa et al., 2016), isópodos (Salomon, 2011), escorpiones (Lira \& Costa, 2014), coleópteros, himenópteros e inclusive otros arácnidos venenosos como Loxosceles laeta (Taucare-Rios \& Canals, 2015). Debido a que las hembras adultas son de mayor tamaño que los machos, estas tienen la capacidad de depredar mamíferos pequeños como ratones (D'Amour et al., 1936), ratas (Nyffeler \& Vetter, 2018), además existen reportes de depredación de anfibios y reptiles (Neill, 1948), como el eslizones (Oshea \& Kelly, 2007; Metcalfe \& Ridgeway, 2013; Shine \& Tamayo, 2016), lacertilios, geckos (Hódar \& Sánchez-Piñero, 2002) y colúbridos (Rocha et al., 2017).

Por su parte, Ninia diademata (Baird \& Girard, 1853), es un pequeño colúbrido (Serpentes: Colubridae) que puede medir hasta $35 \mathrm{~cm}$ de longitud total (Universidad Francisco Marroquín, 2007), con una distribución geográfica que abarca desde el Sureste de México (estados de Chiapas, Hidalgo, Oaxaca, Querétaro, San Luis Potosí, Tabasco y Veracruz), hasta el norte de Centroamérica (Wallach et al., 2014). Debido a sus hábitos semifosoriales, esta especie habita bajo rocas y hojarasca y sale 
a la superficie del suelo para alimentarse (Burger \& Werler, 1954) de pequeños invertebrados como caracoles (Zaher et al., 2014) y babosas (Taylor, 1949). El género Ninia ha sido reportado como presa de serpientes ofiófagas del género Micrurus (Greene, 1976), la culebra perico mexicana Leptophis mexicanus (Henderson \& Hoevers, 1977), y por Bothrops asper (Carbajal-Márquez et al., 2019). Durante la madrugada del lunes 29 de junio de 2020 , alrededor de las 03:50 am, en la localidad de Río Blanco, Veracruz, México (18.831996 ${ }^{\circ} \mathrm{N}, 97.118774^{\circ} \mathrm{O}$, WGS 84; elevación: $1234 \mathrm{msnm}$ ) se observó un ejemplar de Ninia diademata de aproximadamente $10 \mathrm{~cm}$ de longitud total, atrapado sin vida en la telaraña de una hembra de viuda negra de la especie Latrodectus mactans. La telaraña del arácnido se encontraba ubicada en el patio trasero de una vivienda, entre un armario para insumos de jardinería y una ventana. Durante el registro, el arácnido se mantuvo por 2 min posado en su telaraña por debajo de su presa (Fig. 1). Posteriormente, la araña se dirigió hacia la parte superior de la telaraña para refugiarse en una maceta vacía.

Se ha reportado que las arañas del género Latrodectus se alimentan principalmente de insectos, sin embargo, en Brasil,

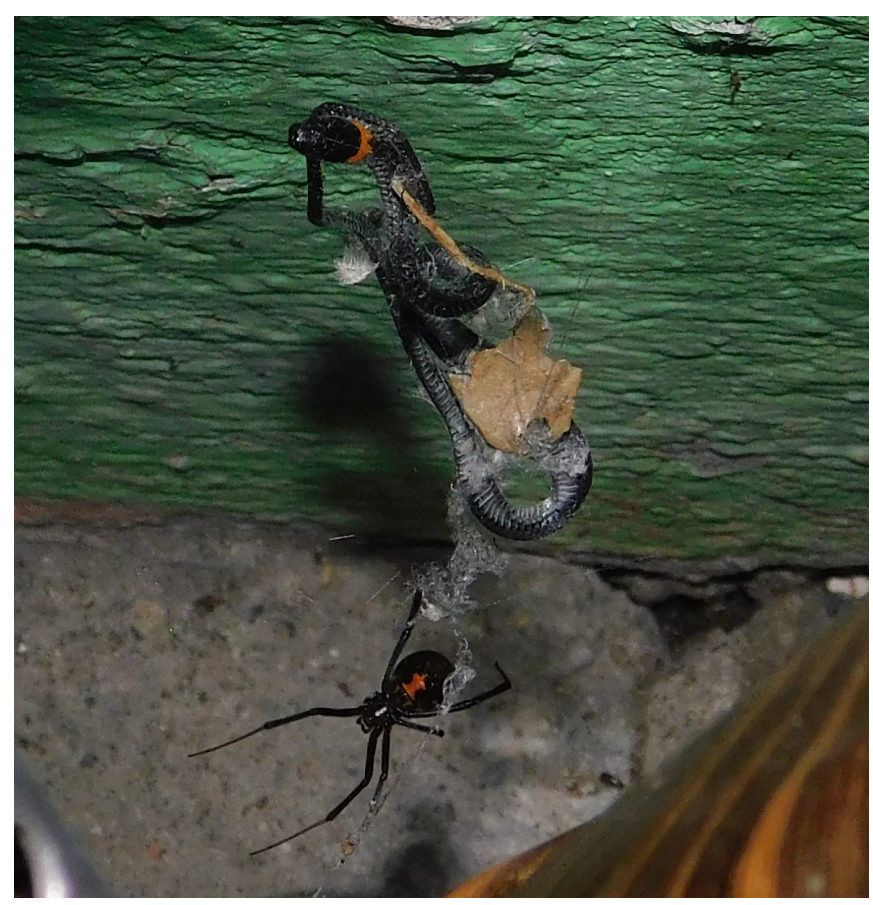

Figure 1. Ninia diademata caught in the web of an adult female of Latrodectus mactans in Río Blanco, Veracruz. Photo by Angel Eduardo Carrasco González.

Figura 1. Ninia diademata atrapada en la red de una hembra adulta de Latrodectus mactans en Río Blanco, Veracruz. Foto de Angel Eduardo Carrasco González.
Rocha et al. (2017) reportaron la depredación de una culebra de cabeza negra (Tantilla melanocephala) por parte de una viuda café (Latrodectus geometricus).

Ninia diademata es un colúbrido que tiene registros históricos de distribución en la Región de las Altas Montañas (Dunn, 1935), mientras que Latrodectus mactans es una especie de arácnido que se distribuye en todos los estados de México (Salcedo-Sánchez et al., 2017), esto revela que la coincidencia de estas especies de serpientes consumidas por arañas del género Latrodectus, es un indicador de que la dieta de este grupo de artrópodos está estrechamente relacionada con la distribución geográfica de la serpientes que concurre en el área en que cohabitan (AguilarLópez et al., 2014) y es un ejemplo de la importancia de los arácnidos sobre la dinámica poblacional de serpientes.

\section{LITERATURA CITADA}

Aguilar-López, J.L., E. Pineda \& R. Luría-Manzano. 2014. Depredación de tres especies de herpetozoos por arañas en la región tropical de Veracruz, México. Revista Mexicana de Biodiversidad 85:965-968.

Burger, W.L. \& J.E. Werler. 1954. The subspecies of Ring-necked Coffee Snake, Ninia diademata, and a Short Biological and Taxonomic Account of the Genus. University of Kansas Science Bulletin 36:643-672.

Carbajal-Márquez, R.A., C.M. García-Balderas, T. RamírezValverde, J.R. Cedeño-Vázquez \& N.G. Blanco-Campos. 2019. New prey items in the diet of snakes from the Yucatán Peninsula, Mexico. Cuadernos de Herpetología 33: 71-74.

Dunn, E.R. 1935. The snakes of the genus Ninia. Proceedings of the National Academy of Sciences of the United States of America 21: 9-12.

D'Amour, F.E., F.E. Becker \& W. Van Riper. 1936. The Black Widow Spider. The Quarterly Review of Biology 11:123-160.

Gaiarsa, M.P., L.R. Vieira de Alencar, C.J. Dias \& M. Martins. 2012. Predator or prey? Predatory interactions between the frog Cyclorampus boraceiensis and the spider Trechaleoides biocellata in the Atlantic forest of southeastern Brazil. Herpetology Notes 5:67-68.

Gonçalo, M.R., H. Couto, \& F. Andreone.2016. Clash of the Venomous: Predation of a Centipede cormocephalus (Chilopoda: 
Scolopendridae) by a Black Widow Latrodectus (Araneae: Theridiidae) in Madagascar. Arachnology 17:58-60.

Greene, H.W. 1976. Scale Overlap, a Directional Sign Stimulus for Prey Ingestion by Ophiophagous Snakes 1. Zeitschrift für Tierpsychologie 41:113-120.

Henderson, R.W. \& L.G. Hoevers. 1977. The seasonal incidence of snakes at a locality in northern Belize. Copeia 1977: 349-355.

Hódar, J.A. \& F. Sánchez-Piñero. 2002. Feeding habits of the blackwidow spider Latrodectus lilianae (Araneae: Theridiidae) in an arid zone of south-east Spain. Journal of Zoology 257:101-109.

Lira, A.F.A. \& A.A. Costa. 2014. First record of a brown widow spider Latrodectus geometricus Koch, 1841 (Araneae, Theridiidae) feeding scorpion (Scorpiones, Bothriuridae) in a Brazilian Atlantic forest. Brazilian Journal of Biology 74:1011.

Metcalfe, D.C. \& P.A. Ridgeway. 2013. A case of web entanglement and apparent predation of the skink Lampropholis delicata (De Vis, 1888) (Sauria: Scincidae: Lygosominae) by the red-back spider Latrodectus hasseltii Thorell, 1870 (Aranea [sic]: Araneomorpha: Theridiidae) in an autochthonous mesic habitat in coastal southeast Australia. Herpetology Notes 6:375-377.

Neill, W.T. 1948. Spiders preying on reptiles and amphibians. Herpetologica 4:158.

Nyffeller, M., D.A. Dean \& W.L. Sterling. 1988. The southern black widow spider, Latrodectus mactans (Araneae, Theridiidae), as a predator of the red imported fire ant, Solenopsis invicta (Hymenoptera, Formicidae), in Texas cotton fields. Journal of Applied Entomology 106:52-57.

Nyffeler, M. 1999. Prey Selection of Spiders in the Field. The Journal of Arachnology 27:317-324

Nyffeler, M. \&Vetter, R.S. 2018. Black widow spiders, Latrodectus spp. (Araneae: Theridiidae), and other spiders feeding on mammals. The Journal of Arachnology 46:541-548.

O'Shea, M. \& K. Kelly. 2017. Predation on a Weasel Skink (Saproscincus mustelinus) (Squamata: Scincidae: Lygosominae) by a Redback Spider (Latrodectus hasselti) (Araneae: Araneomorpha: Theridiidae), with a review of other Latrodectus predation events involving squamates. Herpetofauna 44: 49-55.
Redtox. 2020. Viuda negra / araña capulina. https://www.redtox. org/lo-que-debes-saber/ejemplares-venenosas/aracnidos/ item/96-viuda-negra-arana-capulina. [Consultado el 03/07/20).

Rocha, C.R., P.C. Motta, A. de Souza Portella, M. Saboya \& R. Brandão. 2017. Predation of the snake Tantilla melanocephala (Squamata: Colubridae) by the spider Latrodectus geometricus (Araneae: Theridiidae) in Central Brazil. Herpetology Notes 10:647-650.

Salceda-Sánchez, B., V. Hernández-Hernández, E. Conde-Sánchez, M. Vargas-Olmos, J. López-Cárdenas \& H. Huerta. 2017. New Registers de the Latrodectus Walckenaer y Loxosceles Heineken y Lowe Genera and their Distribution in Mexico. Southwestern Entomologist 42: 575-582.

Salomon, M. 2011. The natural diet of a polyphagous predator, Latrodectus hesperus (Araneae: Theridiidae), over one year. The Journal of Arachnology 39:154-160.

Shine, R. \& B. Tamayo. 2016. When predators become prey: the lizard-eating spiders of suburbia. Australian Zoologist 38:212213.

Taucare-Ríos, A. \& M. Canals. 2015. Feeding habits of the brown widow spider Latrodectus geometricus (Araneae: Theridiidae) in northern Chile. Revista Iberica de Aracnología 27:155-158.

Taylor, E.H. 1949. A preliminary account of the herpetology of the state of San Luis Potosi, México. University of Kansas Science Bulletin 33:169-215.

Universidad Francisco Marroquín. 2007. Arboretum. https:// arboretum.ufm.edu/animales/ninia-diademata. [Consultado el 01/07/2020]

Wallach, V., K.L. Williams \& J. Boundy. 2014. Snakes of the world: a catalogue of living and extinct species. CRC Press, Taylor \& Francis Group. Boca Raton, Florida, E.U.

World Spider Catalog. 2019. Latrodectus mactans. http://wsc.nmbe.ch [Consultado el 17/03/2019]

Zaher, H., L. de Oliveira, F.G. Grazziotin, M. Campagner, C. Jared, M.M. Antoniazii \& A.L. Prudente. 2014. Consuming viscous prey: A novel protein-secreting delivery system in neotropical snaileating snakes. Evolutionary Biology 14:1-28. 